\title{
Suppression of RRM2 inhibits cell proliferation, causes cell cycle arrest and promotes the apoptosis of human neuroblastoma cells and in human neuroblastoma RRM2 is suppressed following chemotherapy
}

\author{
JUNFENG LI $^{1}$, JINGLIN PANG ${ }^{2}$, YONGDONG LIU ${ }^{1}$, JING ZHANG ${ }^{1}$, \\ CHUANGUANG ZHANG ${ }^{1}$, GANG SHEN ${ }^{1}$ and LILI SONG ${ }^{1}$ \\ Departments of ${ }^{1}$ Pediatric Surgery and ${ }^{2}$ Health Management Center, Weifang People's Hospital, \\ Weifang, Shandong 261041, P.R. China
}

Received July 20, 2017; Accepted December 4, 2017

DOI: $10.3892 / o r .2018 .6420$

\begin{abstract}
Ribonucleotide reductase regulatory subunit M2 (RRM2) is a rate-limiting enzyme for DNA synthesis and repair. RRM2 has vital roles in controlling the progression of cancer. In the present study, we investigated the RRM2 level in neuroblastoma tissues, analyzed its relationship with clinicopathological characteristics of neuroblastoma patients, and explored the effect of RRM2 on the biological functions of neuroblastoma cells. RRM2 levels in 67 pairs of neuroblastoma and matched adjacent non-cancerous tissues were detected by qRT-PCR, and its association with patient clinicopathological features was assessed. Using RRM2 siRNA, the role of RRM2 in cell viability was detected by CCK- 8 assay, and the effects on cell cycle distribution and cell apoptosis were detected by flow cytometry. Hoechst 33342 staining was also performed. For RRM2 protein detection in cells and tissues, western blot analyses were employed. Our results revealed that RRM2 expression was significant higher in neuroblastoma tissues than that noted in adjacent non-cancerous tissues at both the mRNA and protein levels. The increased RRM2 level was significantly associated with clinical stage. RRM2 levels were suppressed in stage III and IV tumors in the chemotherapy subgroup, compared with levels noted in tumors in the preoperative non-chemotherapy subgroup. RRM2 siRNA significantly inhibited cell viability in the SH-5Y5Y cells, induced cell arrest in the G0/G1 phase, and enhanced cell apoptosis. Taken together, overexpression of RRM2 is associated with the genesis and progression of neuroblastoma, and may be a potential chemotherapeutic target.
\end{abstract}

Correspondence to: Dr Lili Song, Department of Pediatric Surgery, Weifang People's Hospital, 151 Guangwen Street, Kuiwen, Weifang, Shandong 261041, P.R. China

E-mail: sn0021@163.com; 943169039@qq.com

Key words: RRM2, neuroblastoma, chemotherapy, biological function

\section{Introduction}

Neuroblastoma, one of the most common extracranial solid tumors, accounts for $\sim 10 \%$ of all childhood cancers (1). Moreover, neuroblastoma is the most frequently diagnosed neoplasm during infancy (1). It is known that neuroblastoma originates from embryonal neural crest cells that play an important role in sympathetic nervous system development (2). Neuroblastoma is a very heterogeneous and incurable tumor, ranging from the presentation of spontaneously regressing growth to aggressive malignant potential $(3,4)$. It was reported that the clinical outcome of neuroblastoma is closely correlated with patient age, tumor stage and histological classification $(5,6)$. Although huge advances have been achieved in neuroblastoma treatment, such as surgery and chemotherapy, its morbidity and mortality remain at a high level $(4,5)$. Thus far, the elusive molecular mechanism underlying the genesis and progression of neuroblastoma remain unclear.

Overexpression of the catalytic subunit (RRM2) of ribonucleotide reductase is involved in the modification of gemcitabine metabolism, and thus induces inherent or acquired resistance to chemotherapeutic agents such as gemcitabine (7). Ribonucleotide reductase (RR) catalyzes the inhibition of ribonucleotides yielding deoxyribonucleotides, and is a rate-limiting enzyme for DNA synthesis (8). Transcriptional regulation is the main mechanism in controlling the enzymatic activity of RR (9). RRM2 expression at the mRNA and protein levels were found to be increased 9- and 2-fold in the gemcitabine-resistant cell line KB-Gem, respectively (10). In addition, it was demonstrated that RRM2 expression levels in tumors are a potential predictive indicator of treatment responsiveness to chemotherapeutic agents (11). Knockdown of RRM2 (12) or treatment with flavopiridol (a cyclin-dependent kinase inhibitor) (13) rescued the sensitivity of cancer cells to chemotherapeutic agents. Flavopiridol promotes cell apoptosis by gemcitabine in human pancreatic, gastric and colon cancer cells, which may be associated with inhibition of the RRM2 protein (13). 
Although the RRM2 levels in various tumor types have been investigated, little is known concerning the level and role of RRM2 in neuroblastoma. In the present study, we assessed the RRM2 levels in human neuroblastoma tissues and matched adjacent non-cancerous tissues and the correlation between the RRM2 levels in neurobastoma and various clinicopathological characteristics. In addition, the effect of chemotherapy on RRM2 expression, and the role of RRM 2 in the biological functions of neuroblastoma cells were also explored.

\section{Materials and methods}

Patients and specimens. Neuroblastoma specimens were collected from 67 children (including 29 males and 38 females, ranging in age from 1 month to 13 years with a median age of 5.16 years) with primary neuroblastoma during surgical operation at the Department of Pediatric Surgery, Weifang People's Hospital (Weifang, China) between September 2014 and August 2016. The pairs of neuroblastoma and matched adjacent non-cancerous tissues were collected from the site $>5 \mathrm{~cm}$ away from the primary site. The present study was approved by the Ethics Review Committee of Weifang People's Hospital and signed informed consent was obtained from all patients. Clinical staging of neuroblastoma was assessed according to the International Neuroblastoma Staging System by two independent pathologic examinations (14). Among the 67 cases of neuroblastoma, 40 cases had not received preoperative treatment including 27 cases of stage I and II and 13 cases of stage III and IV. The other 27 cases received the same chemotherapy before surgery. All specimens were snap frozen in liquid nitrogen and stored at $-80^{\circ} \mathrm{C}$ until use. The clinicopathological characteristics of all 67 neuroblastoma patients are summarized in Table I.

Cell culture and transfection. The neuroblastoma cell line SH-5Y5Y and human neural stem cell line N7800-200 were purchased from the American Type Culture Collection (ATCC; Manassas, VA, USA). SH-5Y5Y cell and N7800-200 were maintained in DMEM/F12 supplementing with $10 \%$ fetal bovine serum (FBS) (both from Gibco, Carlsbad, CA, USA) at $37^{\circ} \mathrm{C}$, in a humidified $5 \% \mathrm{CO}_{2}$ atmosphere. For transfection of RRM2 siRNA, the RRM2 siRNAs (siRNA-1, 5'-GCGAUU UAGCCAAGAAGUUCA-3'; siRNA-2, 5'-GCGAUUUAG CCAAGAAGUUTT-3'; siRNA-3, 5'-GGGAUUAAACAG UCCUUUATT-3'; mixed) and negative control (NC) siRNA (5'-UAGCGACUAAACACAUCAAUU-3') were constructed. Cell transfection was performed using Lipofectamine ${ }^{\mathrm{TM}} 2000$ (Invitrogen, Carlsbad, CA, USA) according to the manufacturer's instructions. After $48 \mathrm{~h}$, the knockdown effects of RRM2 expression were confirmed by qRT-PCR.

qRT-PCR. Quantitative real-time PCR (qRT-PCR) was performed using an ABI 7500 Real-Time PCR System (Applied Biosystems, Foster City, CA, USA) with SYBR Premix (Takara Bio, Otsu, Japan). Primer pairs used for real-time PCR analysis of RRM2 were 5'-CACGGAGCCGAAAACTAA AGC-3' and 5'-TCTGCCTTCTTATACATCTGCCA-3'. The PCR reaction was performed in conditions: Initiation $30 \mathrm{sec}$ at $95^{\circ} \mathrm{C}$, amplification $5 \mathrm{sec}$ of 40 cycles at $95^{\circ} \mathrm{C}$ and $34 \mathrm{sec}$ at $60^{\circ} \mathrm{C}$. The experiments were performed in triplicate. Data was normalized to GAPDH using the $\Delta \Delta \mathrm{Ct}$ method.
Table I. Correlation between RRM2 and clinicopathological parameters of the neuroblastoma patients.

\begin{tabular}{lccc}
\hline Characteristics & $\begin{array}{c}\text { No. of } \\
\text { cases }\end{array}$ & $\begin{array}{c}\text { Expression of } \\
\text { RRM2 (fold) }\end{array}$ & P-value \\
\hline $\begin{array}{l}\text { Sex } \\
\text { Male }\end{array}$ & 29 & $2.512 \pm 0.104$ & \\
Female & 38 & $2.934 \pm 0.197$ & 0.190 \\
Age (years) & & & \\
$\geq 5$ & 22 & $2.856 \pm 0.290$ & \\
$<5$ & 45 & $2.016 \pm 0.245$ & 0.542 \\
TNM stage & & & \\
I+II & 27 & $1.462 \pm 0.187$ & 0.016 \\
III+IV & 40 & $2.872 \pm 0.231$ & \\
Histology & & & 0.672 \\
Favorable & 36 & $2.216 \pm 0.105$ & \\
Unfavorable & 31 & $3.010 \pm 0.321$ & \\
$\begin{array}{l}\text { Preoperative } \\
\text { chemotherapy }\end{array}$ & & & \\
Yes & & & \\
No & 27 & $2.421 \pm 0.165$ & \\
\hline
\end{tabular}

RRM2, ribonucleotide reductase M2; TNM, tumor-node-metastasis.

Western blotting. Protein was extracted from tissues using RIPA (15). After centrifugation at 12,000 x g $10 \mathrm{~min}$, protein was collected and its concentration was measured by an enhanced BCA protein assay kit (Beyotime Institute of Biotechnology, Haimen, China). Protein $(30 \mu \mathrm{g})$ of each sample was separated by $10 \%$ SDS-PAGE, and then was transferred onto PVDF membranes (Millipore, Billerica, MA, USA). After being blocked in 5\% non-fat milk for $1 \mathrm{~h}$, the membranes were then incubated with the primary antibody anti-RRM2 (1:1,000; Abcam Biotechnology, Cambridge, UK) overnight at $4^{\circ} \mathrm{C}$. Then, horseradish peroxidase (HRP)-conjugated secondary antibodies were used to incubate the membranes for $1 \mathrm{~h}$ at room temperature. Anti-GAPDH (1:1,000; Santa Cruz Biotechnology, Inc., Santa Cruz, CA, USA) was used as an internal control. The blots were visualized using an enhanced ECL detection system (Thermo Fisher Scientific, Inc., Waltham, MA, USA) and data were analyzed using ImageJ software (NIH, Bethesda, MD, USA).

Cell proliferation. Cells (400/well) were placed in a 6-well plate in triplicate. After transfection, the effect of RRM2 on SH-5Y5Y cell proliferation was performed using the Cell Counting Kit-8 (CCK-8; Beyotime Institute of Biotechnology). In brief, at various time points (1, 2, 3 and 4 days), CCK- 8 (10 $\mu \mathrm{l}$ ) was added to each well at $37^{\circ} \mathrm{C}$ for $1.5 \mathrm{~h}$. Then, the cells were harvest and the absorbance at $450 \mathrm{~nm}$ was detected by a microplate spectrophotometer.

Cell apoptosis and cell cycle. Cell cycle and cell apoptosis were measured using flow cytometry. In brief, after transfection, cells were washed with PBS, trypsinized and resuspended in ice-cold PBS. After centrifugation at $300 \mathrm{x} \mathrm{g}, 5 \mathrm{~min}$, at $4^{\circ} \mathrm{C}$, 

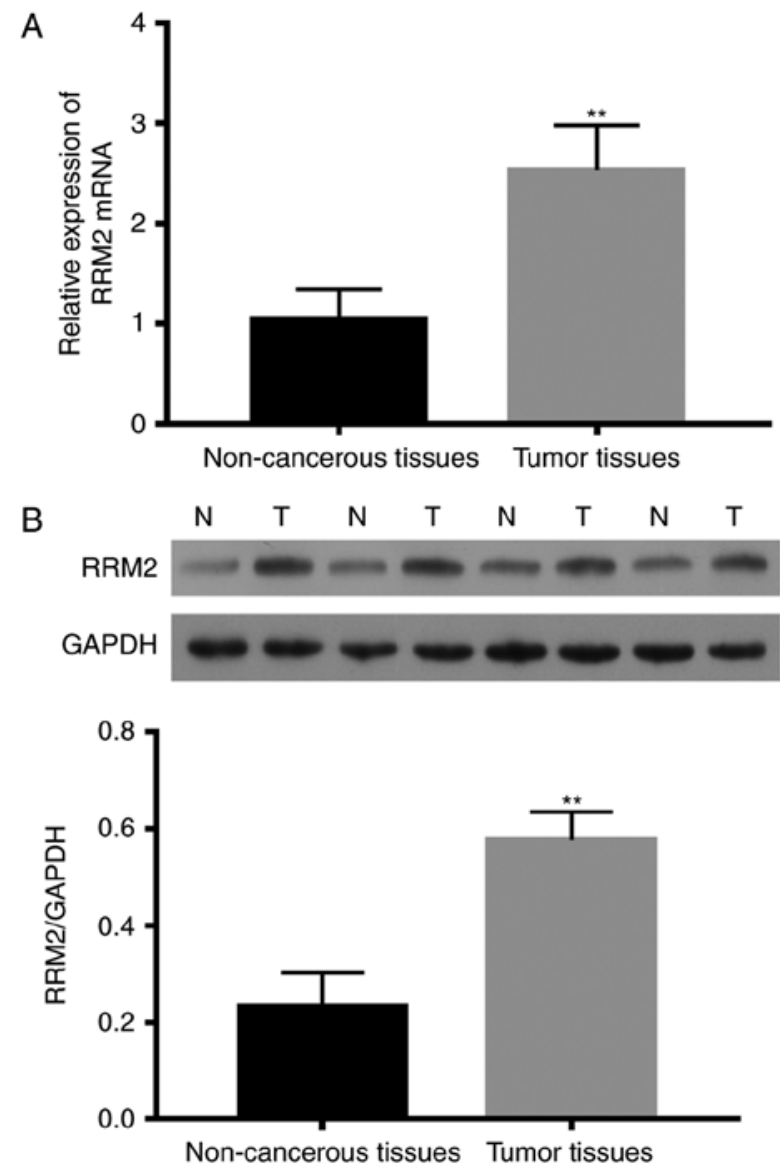

Figure 1. Expression of RRM2 in 67 pairs of neuroblastoma (T) and matched adjacent non-cancerous tissues (N). (A) Overexpression of RRM2 in neuroblastoma tissues was detected by qRT-PCR, compared with non-cancerous tissues. (B) RRM2 protein level was assessed by western blotting and the data were quantified. ${ }^{* *} \mathrm{P}<0.01$ vs. the non-cancerous tissues.

the cells were fixed and permeabilized by $70 \%$ ethanol at $-20^{\circ} \mathrm{C}$. After incubation with a propidium iodide (PI) staining solution $(50 \mu \mathrm{g} / \mathrm{ml}$ PI and $100 \mu \mathrm{g} / \mathrm{ml}$ RNase A in PBS), in the dark for $30 \mathrm{~min}$, the PI fluorescence was measured using flow cytometer (BD Biosciences, Franklin Lakes, NJ, USA). The percentages of cells in the G0/G1, S and G2 phases were analyzed using ModFit software (BD Biosciences).

The harvested cells also underwent apoptosis detection. Cell apoptosis was performed using flow cytometry with the Annexin V-FITC apoptosis detection kit (Sigma, St. Louis, MO, USA). FITC(+) and PI(-) cells represent early apoptotic cells, and FITC(+) and PI(+) cells represent late apoptotic cells. Cell apoptosis was confirmed using Hoechst 33342 staining.

Statistical analysis. Data are presented as mean \pm SD from at least three independent experiments. Differences were compared using SPSS 15.0 statistical software (SPSS, Inc., Chicago, IL, USA) with the Student's t-test and one-way analysis of variance (ANOVA). P-value $<0.05$ was indicative of statistical significance.

\section{Results}

Upregulation of RRM2 in neuroblastoma tissues. RRM2 levels in all 67 pairs of neuroblastoma and adjacent non-cancerous
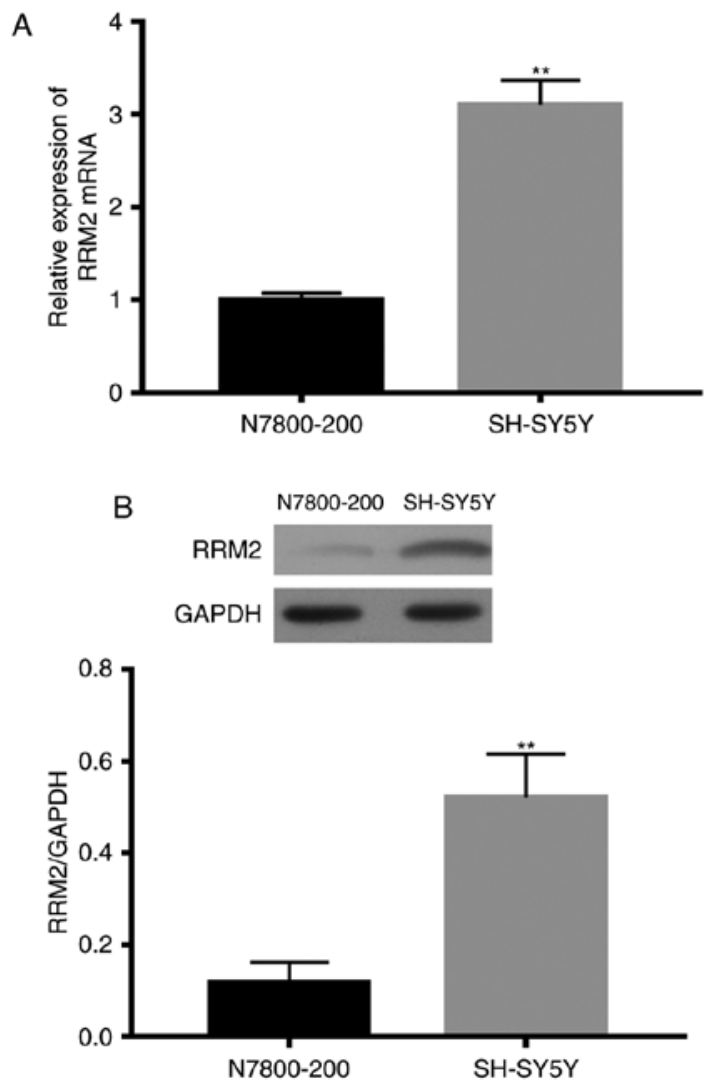

Figure 2. RRM2 expression in SH-5Y5Y cells. (A) RRM2 mRNA expression levels in SH-5Y5Y and N7800-200 cells were detected by qRT-PCR. (B) RRM2 protein level was detected by western blotting and data were quantified; ${ }^{* *} \mathrm{P}<0.01$ vs. N7800-200 cells.

tissues were detected using qRT-PCR and western blotting. Among the 40 patients that did not receive preoperative treatment, RRM2 mRNA expression in the neuroblastoma tissues was significant higher than that noted in the non-cancerous tissues $(\mathrm{P}<0.01)$ (Fig. 1A). The RRM2 protein levels in neuroblastoma tissues were also higher than levels in the non-cancerous tissues (Fig. 1B).

Correlation between RRM2 and clinicopathological characteristics. The RRM2 mRNA level was significantly associated with the clinical stage of the neuroblastoma patients. The RRM2 mRNA expression in stage III and IV neuroblastoma tissues was significant higher than that in stage I and II tissues $(\mathrm{P}=0.016)$ (Table I). There was no significant association between RRM2 mRNA expression and sex, age and histological classification (Table I).

Effect of chemotherapy on RRM2 expression. We investigated the RRM2 expression level after chemotherapy. Results showed that in stage III and IV neuroblastoma tissues, the chemotherapy subgroup (27 cases) expressed lower RRM2 than the preoperative non-chemotherapy subgroup (13 cases) $(\mathrm{P}=0.011)$ (Table I).

RRM2 expression in SH-5Y5Y cells. We detected RRM2 mRNA expression in SH-5Y5Y and N7800-200 cells by qRT-PCR. RRM2 mRNA expression in the SH-5 Y5Y cells was significant higher than that in the N7800-200 cells (Fig. 2A). 
A

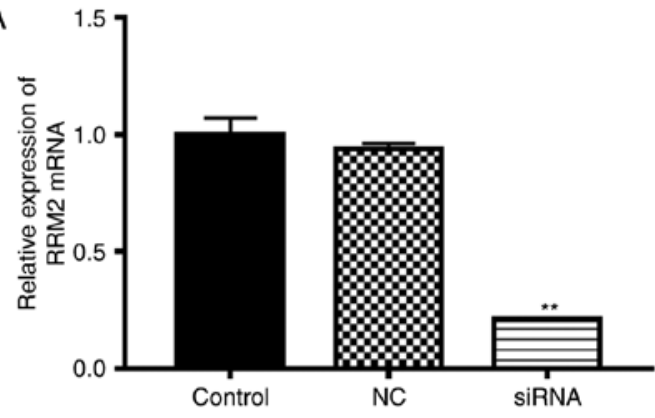

B
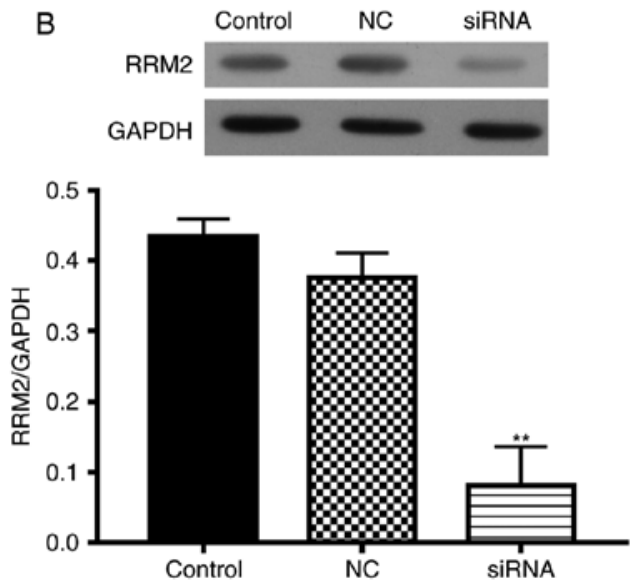

Figure 3. Effect of RRM2 siRNA on RRM2 expression in SH-5Y5Y cells. (A) RRM2 mRNA expression was detected using qRT-PCR; (B) RRM2 protein level was confirmed using western blotting and data were quantified. ${ }^{* *} \mathrm{P}<0.01$ vs. wild-type cells (control).

The RRM2 protein level was measured by western blotting. The RRM2 protein level in SH-5Y5Y cells was also significant higher than that in the N7800-200 cells (Fig. 2B).

RRM2 siRNA inhibits the viability of SH-5Y5Y cells. To investigate the function of RRM2 in SH-5Y5Y cells, we transfected cells with RRM2-siRNA, with non-functional siRNA as the negative control (NC). RRM2 mRNA expression in the RRM2-siRNA transfected cells was less than that in the wild-type group (Fig. 3A). The RRM2 protein level was also inhibited by RRM2 siRNA (Fig. 3B).

The cell viability of the transfected cells was assessed by CCK-8 assay (Fig. 4). At $72 \mathrm{~h}$, cell viability was significantly inhibited by RRM2 siRNA compared with the control and NC groups $(\mathrm{P}<0.05)$.

RRM2 siRNA induces cell cycle arrest in the G0/G1 phase. To determine whether RRM2 siRNA decreases the cell viability by decreasing cell proliferation, the cell cycle was performed by flow cytometry (Fig. 5). The percentage of cells in the G0/G1 phase was significantly increased by RRM2 siRNA compared with the percentage in the control and NC groups $(\mathrm{P}<0.05)$, while the proportion of cells in the $\mathrm{S}$ phase was significantly decreased (Fig. 5A and B). Thus, RRM2 siRNA induced cell arrest in the $\mathrm{G} 0 / \mathrm{G} 1$ phase.

RRM2 siRNA induces cell apoptosis in SH-5Y5Y cells. To further determine whether RRM2 siRNA decreases the cell number by promoting cell apoptosis, cell apoptosis

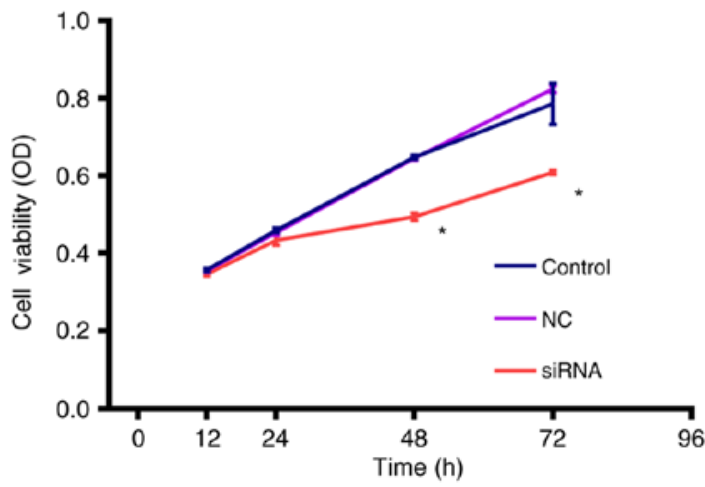

Figure 4. RMP2 siRNA inhibits cell viability. The viable cells at 12, 24, 48 and $72 \mathrm{~h}$ were quantified by CCK- 8 assay. Differences were analyzed by ANOVA; ${ }^{\mathrm{P}}<0.05$ vs. control.

was evaluated using flow cytometry and Hoechst 33342 staining (Fig. 6). The early and late apoptosis rates were significantly increased by RRM2 siRNA compared with the control and NC groups (Fig. 6A and B) $(\mathrm{P}<0.05)$. Similar to the flow cytometry results, Hoechst 33342 staining showed that RRM2 siRNA promoted cell apoptosis (Fig. 6C). Thus, RRM2 siRNA promoted cell apoptosis in the SH-5Y5Y cells.

\section{Discussion}

Ribonucleotide reductase M2 (RRM2), a rate-limiting enzyme for DNA synthesis and repair $(16,17)$, was found to be highly expressed in various diseases including gestational trophoblastic disease, breast, pancreatic and gallbladder cancer, which is related to the growth, invasion and chemoresistance of malignant tumors $(11,18-20)$. In the present study, RRM2 was detected in 67 pairs of neuroblastoma tissues and adjacent non-cancerous tissues. When patients did not receive preoperative treatment, RRM2 expression at the mRNA and protein levels was significant higher in neuroblastoma tissues than that in the adjacent non-cancerous tissues.

It was previously demonstrated that RRM2 is overexpressed in gastric carcinoma tissues compared to that noted in normal gastric mucosa, and it was associated with sex, depth of invasion, EB virus infection, but not with age, tumor size, histological type and lymph node metastasis (21). The high expression of RRM2 in tumor specimens from patients with bladder cancer suggests that RRM2 may be an indicator and potential target of early diagnosis and treatment of bladder cancer (22). In the present study, a high level of RRM2 was significantly associated with the clinical stage in patients with neuroblastoma. The RRM2 mRNA expression in stage III and IV tissues was significant higher than that noted in stage I and II tissues. RRM2 mRNA expression was not associated with sex, age, and histological classification. Thus, RRM2 may a diagnostic indicator and a therapeutic target for neuroblastoma.

It was demonstrated that drug resistance in tumor cells is associated with a prolonged DNA replication phase, and that downregulation of RRM2 expression can increase cell apoptosis induced by chemotherapeutic agents (23). In the present study, tissues from the chemotherapy subgroup had suppressed RRM2 levels in stage III and IV tumors, compared 
A

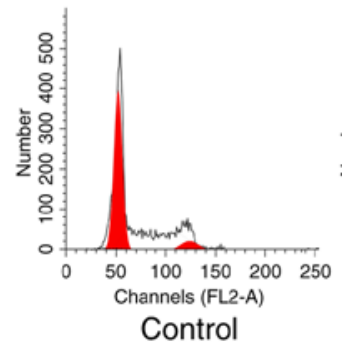

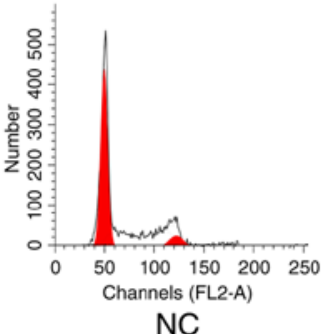

NC
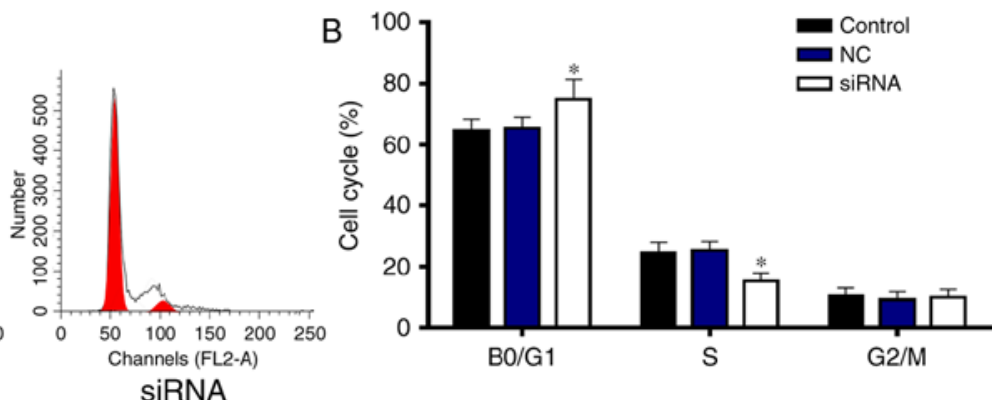

Figure 5. Representative cell cycle analyses by flow cytometry. RRM2 siRNA arrested the cell cycle in the G0/G1 phase. ${ }^{*} \mathrm{P}<0.05$ vs. control.

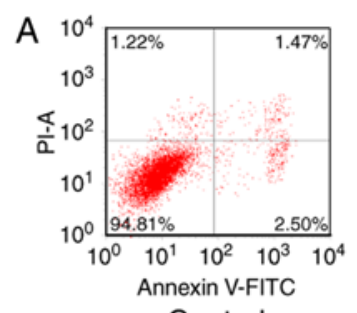

Control

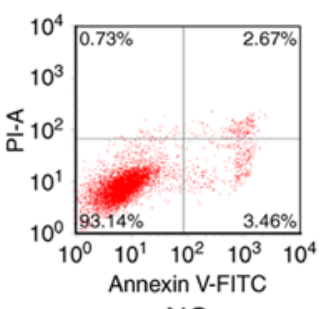

NC

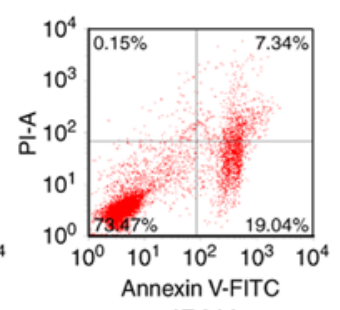

SiRNA

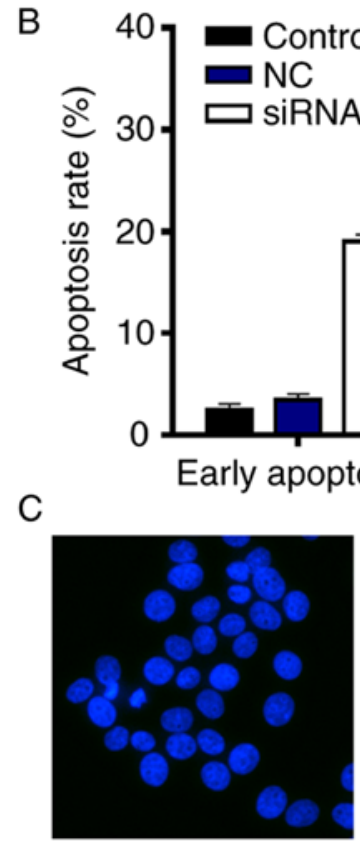

Control

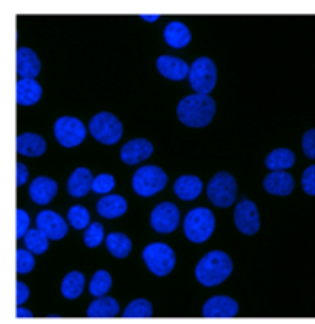

NC

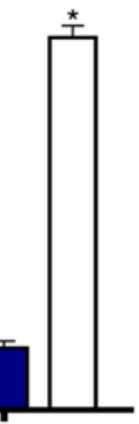

Total apoptosis

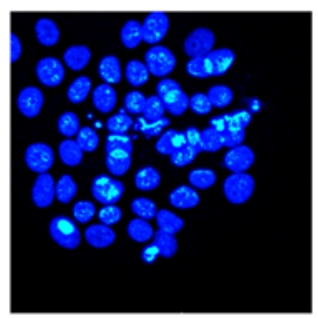

SiRNA

Figure 6. Detection of cell apoptosis using flow cytometry and Hoechst 33342 staining. (A) Flow cytometry, (B) the apoptosis rate and (C) Hoechst 33342 staining. RRM2 siRNA induced cell apoptosis; * $\mathrm{P}<0.05$ vs. control.

with the preoperative non-chemotherapy subgroup, indicating the RRM2 may be associated with chemotherapy. The effects of the reduction or blocking of RRM2 expression levels on the proliferation and apoptosis of SH-5Y5Y cells exposed to chemotherapy drugs may be evaluated in the future.

A high level of RRM2 is closely correlated with increased resistance to chemotherapy in cancer cells, and the reduction or blocking of the RRM2 expression levels by various techniques can improve the sensitivity to chemotherapeutic agents in cancer cells $(12,24)$. At present, the method used for suppression of the expression level of RRM2 mainly includes antisense oligonucleotides or specific drugs $(25,26)$. Recently, studies have reported that blocking RRM2 expression by RNAi may be a new strategy for the gene therapy of malignant tumors $(27,28)$.

Studies have found that cancer cells with a high RRM2 expression level have induced VEGF mRNA expression, which then confers cancer cells with increased growth, invasion and metastasis characteristics, and thus a poor prognosis (18). RRM2 serves as a rate-limiting enzyme for DNA synthesis, and its role is closely related to cell survival $(29,30)$. We detected the RRM2 levels in SH-5Y5Y and N7800-200 cells. The results found that the RRM2 mRNA and protein levels in the SH-5Y5Y cells were significant higher than levels in the 
N7800-200 cells, confirming the overexpression of RRM2 in neuroblastoma cells. To explore the biological role of RRM2 in SH-5Y5Y cells, we transfected cells with RRM2-siRNA or negative control non-functional siRNA (NC). The cell viability was significantly inhibited by RRM2 siRNA compared with the control and NC groups. RRM2 siRNA induced cell cycle arrest in the G0/G1 phase, suggesting that RRM2 siRNA decreased the cell number by decreasing proliferation. RRM2 siRNA promoted cell apoptosis in the SH-5Y5Y cells, suggesting that RRM2 siRNA also decreased the cell number by promoting cell apoptosis. The research showed that there is a direct correlation between RRM 2 and tumor biological behavior. Its high expression has clinical value for early diagnosis and treatment of malignant tumors. Therefore RRM2 is expected to become a new index for malignant tumor diagnosis and prognostic evaluation.

In conclusion, the RRM2 level in neuroblastoma tissues was found to be correlated with clinical stage, and its overexpression was suppressed by chemotherapy. Knockdown of RRM2 decreased cell viability, induced cell cycle arrest in the G0/G phase and promoted cell apoptosis. Our findings suggest that RRM2 may play a vital role in the progression of neuroblastoma and could be a promising therapeutic target.

\section{References}

1. Matthay KK, Maris JM, Schleiermacher G, Nakagawara A, Mackall CL, Diller L and Weiss WA: Neuroblastoma. Nat Rev Dis Primers 2: 16078, 2016.

2. Marshall GM, Carter DR, Cheung BB, Liu T, Mateos MK, Meyerowitz JG and Weiss WA: The prenatal origins of cancer. Nat Rev Cancer 14: 277-289, 2014.

3. Takahashi Y, Sipp D and Enomoto H: Tissue interactions in neural crest cell development and disease. Science 341: 860-863, 2013.

4. Maris JM, Hogarty MD, Bagatell R and Cohn SL: Neuroblastoma. Lancet 369: 2106-2120, 2007.

5. Maris JM: Recent advances in neuroblastoma. N Engl J Med 362: 2202-2211, 2010.

6. Cheung NK and Dyer MA: Neuroblastoma: Developmental biology, cancer genomics and immunotherapy. Nat Rev Cancer 13: 397-411, 2013

7. Bergman AM, Pinedo HM and Peters GJ: Determinants of resistance to 2',2'-difluorodeoxycytidine (gemcitabine). Drug Resist Updat 5: 19-33, 2002.

8. Lewis CS, Voelkel-Johnson C and Smith CD: Suppression of c-Myc and RRM2 expression in pancreatic cancer cells by the sphingosine kinase-2 inhibitor ABC294640. Oncotarget 7: 60181-60192, 2016.

9. Eriksson S and Martin DW Jr: Ribonucleotide reductase in cultured mouse lymphoma cells. Cell cycle-dependent variation in the activity of subunit protein M2. J Biol Chem 256: 9436-9440, 1981.

10. Goan YG, Zhou B, Hu E, Mi S and Yen Y: Overexpression of ribonucleotide reductase as a mechanism of resistance to 2,2-difluorodeoxycytidine in the human KB cancer cell line. Cancer Res 59: 4204-4207, 1999.

11. Itoi T, Sofuni A, Fukushima N, Itokawa F, Tsuchiya T, Kurihara T, Moriyasu F, Tsuchida A and Kasuya K: Ribonucleotide reductase subunit M2 mRNA expression in pretreatment biopsies obtained from unresectable pancreatic carcinomas. J Gastroenterol 42: 389-394, 2007.

12. Duxbury MS, Ito H, Zinner MJ, Ashley SW and Whang EE: RNA interference targeting the M2 subunit of ribonucleotide reductase enhances pancreatic adenocarcinoma chemosensitivity to gemcitabine. Oncogene 23: 1539-1548, 2004.
13. Jung CP, Motwani MV and Schwartz GK: Flavopiridol increases sensitization to gemcitabine in human gastrointestinal cancer cell lines and correlates with down-regulation of ribonucleotide reductase M2 subunit. Clin Cancer Res 7: 2527-2536, 2001.

14. Brodeur GM, Pritchard J, Berthold F, Carlsen NL, Castel V, Castelberry RP, De Bernardi B, Evans AE, Favrot M, Hedborg F, et al: Revisions of the international criteria for neuroblastoma diagnosis, staging and response to treatment. Prog Clin Biol Res 385: 363-369, 1993.

15. Zeng Y, Yao X, Chen L, Yan Z, Liu J, Zhang Y, Feng T, Wu J and Liu X: Sphingosine-1-phosphate induced epithelial-mesenchymal transition of hepatocellular carcinoma via an MMP-7/syndecan-1/TGF- $\beta$ autocrine loop. Oncotarget 7: 63324-63337, 2016.

16. Reece SY and Seyedsayamdost MR: Long-range proton-coupled electron transfer in the Escherichia coli class Ia ribonucleotide reductase. Essays Biochem 61: 281-292, 2017.

17. Pai CC and Kearsey SE: A critical balance: dNTPs and the maintenance of genome stability. Genes 8: pii: E57, 2017.

18. Cui JQ, Shi YF, Zhou HJ and Li JQ: The changes of gene expression profiles in hydatidiform mole and choriocarcinoma with hyperplasia of trophoblasts. Int J Gynecol Cancer 14: 984-997, 2004.

19. Okamura H, Kamei T, Sakuma N, Hanai N and Ishihara T: Ribonucleotide reductase immunoreactivity in adenocarcinoma cells and malignant or reactive mesothelial cells in serous effusions. Acta Cytol 47: 209-215, 2003.

20. Juhasz A, Vassilakos A, Chew HK, Gandara D and Yen Y: Analysis of ribonucleotide reductase M2 mRNA levels in patient samples after GTI-2040 antisense drug treatment. Oncol Rep 15: 1299-1304, 2006.

21. Morikawa T, Hino R, Uozaki H, Maeda D, Ushiku T, Shinozaki A, Sakatani T and Fukayama M: Expression of ribonucleotide reductase M2 subunit in gastric cancer and effects of RRM2 inhibition in vitro. Hum Pathol 41: 1742-1748, 2010.

22. Morikawa T, Maeda D, Kume H, Homma Y and Fukayama M: Ribonucleotide reductase M2 subunit is a novel diagnostic marker and a potential therapeutic target in bladder cancer. Histopathology 57: 885-892, 2010.

23. Mannargudi MB and Deb S: Clinical pharmacology and clinical trials of ribonucleotide reductase inhibitors: Is it a viable cancer therapy? J Cancer Res Clin Oncol 143: 1499-1529, 2017.

24. Duxbury MS, Ito H, Benoit E, Zinner MJ, Ashley SW and Whang EE: Retrovirally mediated RNA interference targeting the M2 subunit of ribonucleotide reductase: A novel therapeutic strategy in pancreatic cancer. Surgery 136: 261-269, 2004.

25. Fujita H, Ohuchida K, Mizumoto K, Itaba S, Ito T, Nakata K, Yu J, Kayashima T, Souzaki R, Tajiri T, et al: Gene expression levels as predictive markers of outcome in pancreatic cancer after gemcitabine-based adjuvant chemotherapy. Neoplasia 12: 807-817, 2010.

26. Souglakos J, Boukovinas I, Taron M, Mendez P, Mavroudis D, Tripaki M, Hatzidaki D, Koutsopoulos A, Stathopoulos E, Georgoulias V and Rosell R: Ribonucleotide reductase subunits $\mathrm{M} 1$ and M2 mRNA expression levels and clinical outcome of lung adenocarcinoma patients treated with docetaxel/gemcitabine. $\mathrm{Br}$ J Cancer 98: 1710-1715, 2008.

27. Moorthy NS, Cerqueira NM, Ramos MJ and Fernandes PA: Development of ribonucleotide reductase inhibitors: A review on structure activity relationships. Mini Rev Med Chem 13: 1862-1872, 2013.

28. Cerqueira NM, Pereira S, Fernandes PA and Ramos MJ: Overview of ribonucleotide reductase inhibitors: An appealing target in anti-tumour therapy. Curr Med Chem 12: 1283-1294, 2005.

29. Giannattasio M and Branzei D: S-phase checkpoint regulations that preserve replication and chromosome integrity upon dNTP depletion. Cell Mol Life Sci 74: 2361-2380, 2017.

30. Dyawanapelly S, Kumar A and Chourasia MK: Lessons learned from gemcitabine: Impact of therapeutic carrier systems and Gemcitabine's drug conjugates on cancer therapy. Crit Rev Ther Drug Carrier Syst 34: 63-96, 2017. 